\title{
A Randomized, Double-Blind Clinical Trial for Effect of Wisconsin Ginseng (Panax quinquefolius) in Improvement of Rheumatoid Arthritis Associated Fatigue
}

\author{
Vandenhouten Eric Eugene $e^{1,2, \dagger, *}$, Song Xinwei ${ }^{3,4,5,6,7,8 \dagger, *}$ \\ ${ }^{1}$ International Education College, Zhejiang Chinese Medical University, Hangzhou, China \\ ${ }^{2}$ Ginseng Board of Wisconsin Medical Research Committee, Marathon, USA \\ ${ }^{3}$ The First School of Medicine, Zhejiang Chinese Medical University, Hangzhou, China \\ ${ }^{4}$ Department of Rheumatism and Immunology, Zhejiang Provincial Hospital of Chinese Medicine, Hangzhou, China \\ ${ }^{5}$ Rheumatism Professional Committee of Zhejiang Association of TCM, Hangzhou, China \\ ${ }^{6}$ Rheumatism Professional Committee of Zhejiang Association of Integrative Medicine, Hangzhou, China \\ ${ }^{7}$ Rheumatism Professional Committee of Zhejiang Medical Association, Hangzhou, China \\ ${ }^{8}$ Rheumatism Professional Committee of Zhejiang Medical Doctors Association, Hangzhou, China
}

Email address:

gwgtltd@yahoo.com (V. E. Eugene), sxw1055@163.com (Song Xinwei)

${ }^{*}$ Corresponding author

$\uparrow$ Vandenhouten Eric Eugene and Song Xinwei are co-first authors.

\section{To cite this article:}

Vandenhouten Eric Eugene, Song Xinwei. A Randomized, Double-Blind Clinical Trial for Effect of Wisconsin Ginseng (Panax quinquefolius) in Improvement of Rheumatoid Arthritis Associated Fatigue. International Journal of Chinese Medicine. Vol. 4, No. 4, 2020 , pp. $71-81$. doi: $10.11648 /$ j.ijcm.20200404.11

Received: November 2, 2020; Accepted: November 17, 2020; Published: November 27, 2020

\begin{abstract}
Purpose: To observe and evaluate the clinical efficacy of the Wisconsin ginseng (Panax quinquefolius) herbal botanical adjunctive treatment to improve Rheumatoid Arthritis (RA) fatigue. A secondary aim was to observe RA related biomarkers and evaluate the safety of the treatment. Methods: Eligible adult patients with RA were enrolled between September 2019 and January 2020 at a multisite in a double-blind randomized trial with each intervention lasting 8 weeks with prescription medications, diet, and lifestyle kept constant. Interventions consisted of Wisconsin ginseng in doses of 3,000, or 6,000 $\mathrm{mg} /$ day or placebo given in twice daily dosing over 8 weeks. Primary endpoint outcome measures were changes from baseline at 4 and 8 weeks utilizing the Visual analogue scale to evaluate fatigue severity (VAS-F) and Disease activity score 28-joint count erythrocyte sedimentation rate \& C-reactive protein (DAS28 ESR \& CRP), secondary objectives included The health assessment questionnaire disability index (HAQ-DI), Cyclic adenosine monophosphate (cAMP), and Cyclic guanosine monophosphate (cGMP). Safety parameters included complete blood count (CBC), liver, kidney and function. Results: A total of 17 individuals with well-controlled RA that complained of fatigue completed the study. The safety profiles were unaffected. Kruskal-Wallis test was used to compare the efficacy at 4 weeks and 8 weeks after treatment, there was no significant difference in efficacy between the groups 4 weeks after treatment $(\mathrm{Z}=2.914, \mathrm{P}=0.233)$. After 8 weeks of treatment, there was a significant difference in efficacy between the groups $(Z=6.753, P=0.034)$. Further pair comparison showed that the effect of the high-dose $6,000 \mathrm{mg} /$ day group demonstrated greater efficacy than the control group $(\mathrm{Z}=6.325, \mathrm{P}=0.01)$. Conclusions: Addition of Wisconsin ginseng to conventional therapy in RA with fatigue, thus warrants further investigation to guide the role of Wisconsin ginseng to improve fatigue associated with autoimmune disorders as an adjunct treatment. Trial registration: Chinese Clinical Trials Registry. Identifier: ChiCTR1900026257; Registration Date: 2019-09-28.
\end{abstract}

Keywords: Rheumatoid Arthritis, Fatigue, Panax quinquefolius, Wisconsin Ginseng, Complementary and Integrative Health 


\section{Introduction}

Rheumatoid arthritis (RA) is an autoimmune disorder that occurs in approximately 5 per 1000 people and can inevitably prompt severe joint damage and disability [1]. The disability rate of RA ranks high among the arthritic which occur in multiple-joint on the human body, and the incidence of this kind of arthritis is increasing year by year. The incidence of RA is occult, early diagnosis is difficult, and imaging manifestations occur comparatively late. At the point when RA is identified, the patients are usually at an advanced stage of this disease. RA would lead to multiple-joint dysfunction, disability, debilitating fatigue, lower quality of life, respiratory illness, cardiovascular disease and other comorbidities in patients not receiving intervention, fatigue being a common problem for patients with RA described as 'extreme and persistent tiredness, weakness or exhaustion-mental, physical or both [2-5]. The etiology of RA and associated fatigue is still ambiguous $[6,7]$.

The herbal botanical Wisconsin ginseng (Panax quinquefolius) also known as American ginseng listed in Appendix II of the Convention on International Trade in Endangered Species of Wild Fauna and Flora (CITES) is a prominent Oriental Medicine tonic treatment in East Asian countries for over 1000 years [8]. In the USA Wisconsin ginseng is considered a Natural Product scientific area of The National Center for Complementary and Integrative Health (NCCIH). The NCCIH is 1 of the 27 institutes and centers that make up the National Institutes of Health (NIH) [9]. Wisconsin ginseng according to Oriental Medicine [10] philosophies treating RA of the identification pattern of qi and yin deficiency $[11,12]$. by means of tonifying both qi and yin, cooling fire, calming the body and tranquilize the mind, through the aspect of entering the heart, kidney, and lung channels, standard dosage of $3-6 \mathrm{~g}$ per day [13]. Oriental Medicine has a focus on assessing the effectiveness, feasibility and safety of herbal Botanicals in an effort to diversify non-pharmacological treatment and improve disease outcomes that are not generally considered part of conventional medicine. A systematic review of clinical trials of American and Asian ginseng as a treatment for fatigue reported that, although shown to be safe, may be viable treatments for fatigue in people with chronic illness. The biomedical pharmacological active compounds come from the chemical diversity of ginsenosides believed to act on the central nervous system and to have cortisol-modulating and antioxidant as well as anti-inflammatory properties [14].

Based on preclinical and clinical data we hypothesize Wisconsin ginseng ginsenosides $\mathrm{Rb}^{1}, \mathrm{Rg}^{1}, \mathrm{Re}, \mathrm{Rg}^{3}$ and compound $\mathrm{K}$ could demonstrated positive health benefits, taken in the context of its use adjunct to usual therapy in adults with RA associated fatigue. To the best of our knowledge, this is the first 8 week randomized, double-blind clinical trial of assessing the efficacy of Wisconsin ginseng (Panax quinquefolius) as a therapy for RA associated fatigue.

\section{Materials and Method}

\subsection{Study Design and Participants}

The study utilized a randomized, double-blind, placebo-controlled, and included two 8-week treatment arms vs a placebo arm at three centers in the People's Republic of China (Xihu and Shaxia district in Hangzhou). Participants were recruited via advertisements in university bulletin boards and from patients already attending the Rheumatoid clinic at the Department of Rheumatism and Immunology, Zhejiang Provincial Hospital of Chinese Medicine. Inclusion criteria included: (1) RA patients between the ages of 18 and 70 years of age who satisfied the 2010 American College of Rheumatology/European League Against Rheumatism criteria; (2) History of RA related fatigue; (3) Oriental Medicine identification pattern of qi and yin deficiency. Exclusion criteria: (1) pregnant or nursing with the last 37 months; (2) uncontrollable pain; (3) acute infection within the last 7 days; (4) received ginseng herbal treatment within the last 2 months; (5) received vaccinations within the last 7 days; (6) any laboratory test abnormalities; (7) Any known allergy to the ginseng herb; (8) any RA complications within the last 6 months;

The study received approval by a Zhejiang Chinese Medicine University academic review panel and the Zhejiang Provincial Hospital of Chinese Medicine ethics review board (2019-KL-068-02), all patients gave informed written consent before taking part in the study, research followed guidelines of the Declaration of Helsinki, and the study was registered with Chinese Clinical Trial Registry (ChiCTR) (ChiCTR1900026257).

\subsection{Randomization and Masking}

Two consecutive batches of Wisconsin ginseng and one batch of placebo were used for this study in a 1:1 ratio. Participants were to receive a placebo versus 3,000 , or 6,000 $\mathrm{mg}$ per day of Wisconsin ginseng. The entire daily milligrams dosing were partitioned into twice per day daily dosing to be taken in the morning and mid-afternoon with food. A placebo versus 3,000 , or $6,000 \mathrm{mg}$ per day of Wisconsin ginseng were assigned and a code from a computer-generated randomization list by block randomization (block size 3 ). The randomization was generated via an external statistician using computer-generated random number table list. All packages of Wisconsin ginseng and placebo were indistinguishably identical in appearance, with the code as the sole unique identifier. Each participant was assigned a sequential number according to their sequence of enrollment and received Wisconsin ginseng or placebo labelled with the same numbers In this manner, participants were randomly assigned to receive Wisconsin ginseng or placebo in a 1:1 ratio. Investigators involved in randomization and masking did not participate in any other part of the trial. Allocation was masked from all participants, their guardians, and other different investigators.

An individual otherwise not involved in the investigation performed the blinding. The statistician remained blinded to 
the nature of study and treatment throughout all primary and secondary analyses.

\subsection{Procedures}

The Wisconsin ginseng root were fingerprinted utilizing trace elements, pesticides, and contaminants quality control by means of verified lab test reports which were independently certified authentic by The Ginseng Board of Wisconsin's (GBW) (Wausau, WI, USA) third party certifier (1368222-0, 1361282-0, 1401137-0, 1364115-0, 1364505-0, 1364508-01364511-0, 1364524-01364528-0, 1368247-0, 1376048-0). All batches meet The United States Department of Agriculture (USDA) United States Standards for Grades of Cultivated Ginseng [15], safety tests standards of the USA Environmental Protection Agency (EPA) [16], and adhere to USA state, federal, and international conservation regulations of CITES. The Wisconsin ginseng root was also quality verified in The People's Republic of China (PRC) by the Zhejiang Institute For Food and Drug Control (ZIFDC) test report (JK20161507) results of confirmed ginsenosides $\mathrm{Rb}^{1}$ $1.93 \%\left(\mathrm{C}_{54} \mathrm{H}_{92} \mathrm{O}_{23}\right) ; \mathrm{Rg}^{1}\left(\mathrm{C}_{42} \mathrm{H}_{72} \mathrm{O}_{14}\right)$ and $\mathrm{Re}\left(\mathrm{C}_{48} \mathrm{H}_{82} \mathrm{O}_{18}\right)$ $1.89 \%$. The herbal botanical Wisconsin ginseng intervention was purchased from a GBW licensed distributor in the People's Republic of China (PRC), consisted of pure grounded shaved chips of Wisconsin ginseng or a placebo matching packaged by Good Manufacturing Practices (GMP) and administered to patients with 500-mg opaque capsules.

All randomized participants underwent baseline evaluation consisting of a physical examination, history, and laboratory testing. Participants attended the clinic for baseline evaluation on day 0 and then every 4 weeks for observation (week zero baseline, four, and eight of each treatment arm) throughout the duration of the 8 weeks intervention for a total of 3 visits. Laboratory, and clinical measurements, to assess efficacy and safety of study interventions, these observations were collected at each visit. Also at the beginning, middle and end of the study, participants were provided a symptoms diary to record adverse events (AEs) to report at each visit. Participants were encouraged to maintain their standard lifestyle and prescription medication regimen throughout the study.

\subsection{Endpoints}

The primary endpoint was defined as clinical remission: all main symptoms of experimental observation disappeared, being fatigued utilizing Visual analogue scale to evaluate fatigue severity (VAS-F) [17], joint functional activity returned to normal monitored by The health assessment questionnaire-disability index (HAQ-DI) and blood tests that show low levels of inflammation. The main experimental inflammation indicators (DAS28 ESR, CRP) [18] return to normal levels. Disease remission: DAS28<2.6 (Remission) Obvious effect: all symptoms disappeared or the symptoms mainly observed were eliminated, the basic function of joints was restored, and the patients could participate in normal work. Laboratory results were basically within normal levels. Low disease activity: DAS: $\geq 2.6$ but $\leq 3.2$ (Significant effect) Effective: the main symptoms basically disappeared, the main joint function basically recovered or made significant progress, and the laboratory examination indicators significantly improved. Moderate disease activity: DAS $>3.2$ but $\leq 5.1$. (No effect) None effective: No improvement in any aspect compared with before treatment. High disease activity: DAS28 $>5.1$ (Invalid) Treatment invalid: Worsening of all symptoms and laboratory examination indicators 《Guiding Principles for Clinical Research of New Chinese Medicines》 $[19,20]$. Secondary endpoints were HAQ-DI [21] is a self-completed questionnaire used as a comprehensive measure of functional disability in patients with a wide variety of rheumatic diseases, as our research main aim was to observe fatigue. The exploratory biomarker indicators monitored throughout the study were cyclic adenosine phosphate (cAMP) and cyclic guanosine phosphate (cGMP), which are important second messenger substances in cells, which is widely involved in the regulation of various physiological, biochemical reactions and cellular functions in cells. The safety factors observed were hepatic, renal functions, and AEs monitored from baseline day 0 to week 4 , and 8 for the duration of treatment of all participants.

\subsection{Statistical Analysis}

SPSS20.0 software was used for statistical analysis, the measurement data from normal distribution are expressed by mean and standard deviation, comparison between groups using one-way analysis of variance (ANOVA). Measurement data not subject to normal distribution are expressed as median and percentile. Kruskal-Wallis test was used for comparison between groups, counting data is expressed in frequency and percentage, and Chi-square test was used for comparison between groups.

Repeated measurement ANOVA was used for comparison of repeated measurement data between groups. $\mathrm{P}<0.05$ was considered as the level of statistical significant. Statistical analyses were done by external, independent statisticians.

\subsection{Missing Data}

Our study began in September 2019 and in January 2020 was discontinued due to the Global Public Health Emergency Coronavirus (COVID-19) pandemic leading to our sample size being quite small $(\mathrm{N}=17)$.

\section{Results}

\subsection{Patient Enrollment and Withdrawal}

Of the participants that were invited to visit the clinic for a screening visit to be assessed for their eligibility criteria Thirty-five were identified and enrolled in the study from three different Rheumatology outpatient clinic sites, between September 2019 and January 2020. All analyses were based on data frozen on January 04,2020 . A CONSORT diagram is 
shown as Figure 1. During the study period participants stated lack of time for completion of the study, fifteen participants dropped out during the first 4-week intervention phase. Reasons for attrition were: lost to follow up due to schedule conflicts. Three participants dropped out during the second 8-week intervention phase. Reasons for attrition were: lost to follow up due to schedule conflicts. A total of 17 participants (1 man and 16 women), approximately forty-eight percent of the participants completed all study interventions.

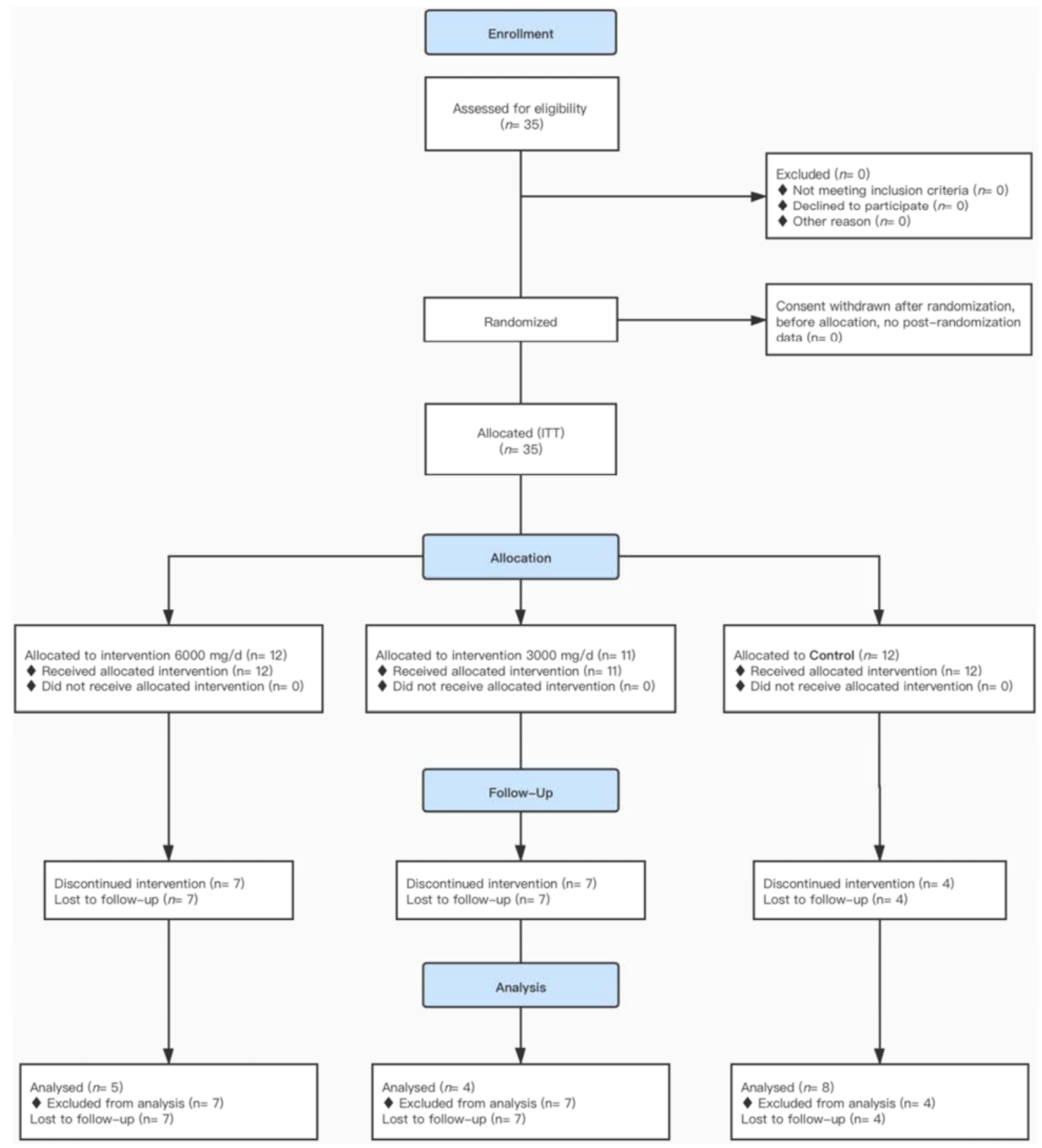

Figure 1. CONSORT diagram flow chart.

\subsection{Baseline Characteristics}

Fisher's Exact Test was used to compare the gender and course of disease among the three groups, one-way ANOVA was used to compare, VAS-F, DAS28 (ESR), DAS28 (CRP), HAQ-DI, cAMP, cGMP, and the age of participants in the three groups. Kruskal-Wallis test was used to compare RF and
CCP of the three groups of samples, the baseline characteristics results of the completed study participants are presented in Table 1, there was no significant difference in the above indicators among the three groups $(\mathrm{P}>0.05)$, indicating comparable baseline among the three groups. 
Table 1. Baseline characteristics of the participants.

\begin{tabular}{|c|c|c|c|c|c|}
\hline Characteristic & Placebo $(\mathbf{N}=8)$ & $3,000 \mathrm{mg}$ Ginseng $(\mathrm{N}=4)$ & $6,000 \mathrm{mg}$ Ginseng $(\mathrm{N}=5)$ & Statistic $\left(\chi^{2} / Z / F\right)$ & $\mathbf{P}$ \\
\hline \multicolumn{6}{|l|}{ Sex } \\
\hline Male & 0 & 1 & 0 & \multirow[t]{2}{*}{2.764} & \multirow[t]{2}{*}{.235} \\
\hline Female & 8 & 3 & 5 & & \\
\hline$<180$ days & 1 & 0 & 0 & \multirow[t]{3}{*}{3.582} & \multirow[t]{3}{*}{.794} \\
\hline $180-360$ days & 0 & 0 & 1 & & \\
\hline$>360$ days & 7 & 4 & 4 & & \\
\hline Age & $50.5 \pm 13.14$ & $51.25 \pm 14.73$ & $47.2 \pm 13.24$ & .126 & .883 \\
\hline $\mathrm{RF}$ & $104.35(69.95,728.35)$ & $236.55(64.85,828.1)$ & $26.5(21.8,57.6)$ & 4.471 & .107 \\
\hline CCP & $179.5(95,200)$ & $39(15.5,129)$ & $78(5,200)$ & 1.196 & .550 \\
\hline DAS28 (ESR) & $5.28 \pm 0.94$ & $5.09 \pm 1.26$ & $5.09 \pm 1.14$ & .068 & .934 \\
\hline DAS28 (CRP) & $4.33 \pm 0.8$ & $4.22 \pm 0.94$ & $3.99 \pm 0.72$ & .275 & .763 \\
\hline HAQ-DI & $0.53 \pm 0.29$ & $0.53 \pm 0.12$ & $0.72 \pm 0.25$ & 1.030 & .382 \\
\hline VAS-F (Energy) & $42.63 \pm 16.76$ & $37.75 \pm 12.74$ & $33.6 \pm 19.68$ & .449 & .647 \\
\hline VAS-F (Fatigue) & $56.97 \pm 16.77$ & $62.59 \pm 12.59$ & $68.23 \pm 18.68$ & .721 & .504 \\
\hline cAMP & $158.23 \pm 22.39$ & $155.33 \pm 14.23$ & $157.58 \pm 15.28$ & .031 & .969 \\
\hline cGMP & $7.44 \pm 1.6$ & $7.17 \pm 1.73$ & $6.65 \pm 1.69$ & .350 & .710 \\
\hline
\end{tabular}

\subsection{Impact of Wisconsin Ginseng on Fatigue}

Repeated measure ANOVA were used to compare VAS-F energy and fatigue of the three groups of participants at different endpoints, the results are shown in Table 2. The intervention and time interaction effect of the VAS-F energy point score were significant $(\mathrm{F}=32.018, \mathrm{P}<0.001)$, Therefore, simple effect analysis was further conducted. The results of simple effect analysis show that without considering the treatment effect, there was no significant difference in VAS-F energy point scores at different endpoint times in the control group $(\mathrm{P}>0.05)$, there were significant differences in VAS-F energy point scores at different endpoints in the $3,000 \mathrm{mg}$ low dose and the $6,000 \mathrm{mg}$ high dose groups, further Pairwise comparison of energy point scores in the low dose and high dose groups showed increased VAS-F energy point scores, as treatment duration increased in time demonstration of a significant increase of point scores was observed; The results of simple effect analysis show that without considering the treatment effect, there was no significant difference in VAS-F energy point scores at week 4 endpoints after treatment within the three groups of participants, at week 8 endpoint after treatment, the VAS-F energy point score of the high dose group was significantly higher than that of the control group.

The intervention and time interaction effect of the VAS-F fatigue point score were significant $(\mathrm{F}=77.23, \mathrm{P}<0.001)$,
Therefore, simple effect analysis was further conducted. The results of simple effect analysis show that without considering the treatment effect, there was no significant difference in VAS-F fatigue point scores at different endpoint times in the control group $(\mathrm{P}>0.05)$, there were significant differences in VAS-F fatigue point scores at different time points in the $3,000 \mathrm{mg}$ low dose and the $6,000 \mathrm{mg}$ high dose groups, further Pairwise comparison of energy point scores in the low dose and high dose groups showed decrease of VAS-F fatigue point scores, as treatment duration increased in time demonstration of a significant decrease of point scores was observed; The results of simple effect analysis show that without considering the treatment effect, there was no significant difference in VAS-F fatigue point scores at week 4 endpoints after treatment within the three groups of participants, at week 8 endpoint after treatment, the VAS-F fatigue point score of the high dose group was significantly lower than that of the control group.

The above results showed that VAS-F point scores in the control group showed no significant change with the duration of treatment, the VAS-F point scores of the low dose group and the high dose group demonstrated increased energy and decreased fatigue significantly with the increase of treatment duration, the intervention effect of the high dose group on VAS-F point scores was significantly better than that of the control group, there was no significant difference between the control group and the low dose group VAS-F point scores.

Table 2. Comparison of fatigue changes using VAS-F after specified treatments.

\begin{tabular}{|c|c|c|c|c|c|c|}
\hline Variable & Group & Week 0 & Week 4 & Week 8 & $\mathbf{F}$ & $\mathbf{P}$ \\
\hline \multirow{5}{*}{ Energy } & Placebo & $42.63 \pm 16.76$ & $44.38 \pm 15.82$ & $45 \pm 15.99$ & 0.574 & .577 \\
\hline & 3,000mg Ginseng & $37.75 \pm 12.74$ & $47.75 \pm 12.74^{\Delta}$ & $57.75 \pm 12.74^{\star}$ & 17.498 & .000 \\
\hline & $6,000 \mathrm{mg}$ Ginseng & $33.6 \pm 19.68$ & $50.2 \pm 12.7 \Delta$ & $68.4 \pm 8.91^{*} \Delta^{\star}$ & 68.336 & .000 \\
\hline & $\mathrm{F}$ & .449 & .263 & 4.663 & & \\
\hline & $\mathrm{P}$ & .647 & .772 & .028 & & \\
\hline \multirow{5}{*}{ Fatigue } & Placebo & $56.97 \pm 16.77$ & $56.44 \pm 16.35$ & $56.97 \pm 15.96$ & .657 & .535 \\
\hline & $3,000 \mathrm{mg}$ Ginseng & $62.59 \pm 12.59$ & $52.59 \pm 12.59^{\Delta}$ & $42.59 \pm 12.59^{\triangle}$ & 30.738 & .000 \\
\hline & $6,000 \mathrm{mg}$ Ginseng & $68.23 \pm 18.68$ & $50.23 \pm 14.7^{\Delta}$ & $32.23 \pm 11.11^{*} \Delta^{*}$ & 124.490 & .000 \\
\hline & $\mathrm{F}$ & .721 & .273 & 4.972 & & \\
\hline & $\mathrm{P}$ & .504 & .765 & .023 & & \\
\hline
\end{tabular}

Note: * indicates significant difference compared with the control group, $\Delta$ indicates significant difference compared with baseline, $\boldsymbol{\square}$ indicates significant difference compared with week 4 endpoint. 


\subsection{Impact of Wisconsin Ginseng on Disease Activity}

Repeated measure ANOVA were used to compare DAS28 (ESR) and DAS28 (CRP) of the three groups of participants at different endpoints, the results are shown in Table 3. The intervention and time interaction effect of the DAS28 (ESR) energy point score were significant $(\mathrm{F}=9.670, \mathrm{P}<0.001)$, therefore, simple effect analysis was further conducted. The results of simple effect analysis show that without considering the treatment effect, there was no significant difference in DAS28 (ESR) scores at different endpoint times in the control group $(\mathrm{P}>0.05)$, there were significant differences in DAS28 (ESR) scores at different endpoints in the $3,000 \mathrm{mg}$ low dose and the $6,000 \mathrm{mg}$ high dose groups, further pairwise comparison of the low dose group at the week 8 endpoint after treatment DAS28 (ESR) scores was significantly lower than the initial baseline pre-treatment and week 4 endpoint after treatment, DAS28 (ESR) scores of high dose group decreased significantly with the increase of treatment time; The results of simple effect analysis show that without considering the treatment effect, there was no significant difference in DAS28 (ESR) scores at week 4 endpoints after treatment within the three groups of participants, at week 8 endpoints after treatment, the DAS28 (ESR) scores of the high dose group was significantly lower than that of the control group.

The intervention and time interaction effect of the DAS28 (CRP) scores were significant $(\mathrm{F}=8.741, \mathrm{P}<0.001)$, therefore, simple effect analysis was further conducted. The results of simple effect analysis show that without considering the treatment effect, there was no significant difference in DAS28 (CRP) scores at different endpoint times in the control group $(\mathrm{P}>0.05)$, there were significant differences in DAS28 (CRP) scores at different endpoints in the $3,000 \mathrm{mg}$ low dose and the $6,000 \mathrm{mg}$ high dose groups, further pairwise comparison of the low dose group at the week 8 endpoint after treatment DAS28 (CRP) scores was significantly lower than the initial baseline pre-treatment and week 4 endpoint after treatment, DAS28 (CRP) scores of high dose group decreased significantly with the increase of treatment time; The results of simple effect analysis show that without considering the treatment effect, there was no significant difference in DAS28 (CRP) scores at week 4 endpoints after treatment within the three groups of participants, at week 8 endpoints after treatment, the DAS28 (CRP) scores of the high dose group was significantly lower than that of the control group.

The above results showed that DAS28 (ESR) and DAS28 (CRP) scores in the control group showed no significant change with the duration of treatment, the ESR and CRP scores of the high dose group decreased significantly as the treatment duration increased, the intervention effect of the high dose group on DAS28 (ESR) and DAS28 (CRP) scores was significantly better than that of the control group, there was no significant difference between the control group and the low dose group DAS28 (ESR) and DAS28 (CRP) scores.

Table 3. Comparison of RA disease activity changes using DAS28 after specified treatments.

\begin{tabular}{|c|c|c|c|c|c|c|}
\hline Variable & Group & Week 0 & Week 4 & Week 8 & $\mathbf{F}$ & $\mathbf{P}$ \\
\hline \multirow{5}{*}{ ESR (DAS28) } & Placebo & $5.28 \pm 0.94$ & $4.92 \pm 0.87$ & $4.93 \pm 0.95$ & 2.581 & .114 \\
\hline & 3,000mg Ginseng & $5.09 \pm 1.26$ & $4.69 \pm 0.89$ & $4.11 \pm 0.85^{\Delta^{\bullet}}$ & 8.015 & .005 \\
\hline & $6,000 \mathrm{mg}$ Ginseng & $5.09 \pm 1.14$ & $3.88 \pm 0.96^{\Delta}$ & $3.22 \pm 0.79^{*} \Delta$ & 31.151 & .000 \\
\hline & $\mathrm{F}$ & .068 & 2.104 & 5.746 & & \\
\hline & $P$ & .934 & .159 & .015 & & \\
\hline \multirow{5}{*}{ CRP (DAS28) } & Placebo & $4.33 \pm 0.8$ & $4.14 \pm 0.77$ & $4.08 \pm 0.77$ & 2.044 & .169 \\
\hline & 3,000mg Ginseng & $4.22 \pm 0.94$ & $3.92 \pm 0.89$ & $3.38 \pm 0.67^{\bullet}$ & 12.422 & .001 \\
\hline & $6,000 \mathrm{mg}$ Ginseng & $3.99 \pm 0.72$ & $3.26 \pm 0.82^{\Delta}$ & $2.7 \pm 0.76^{*} \Delta^{\bullet}$ & 33.214 & .000 \\
\hline & $\mathrm{F}$ & .275 & 1.821 & 5.404 & & \\
\hline & $\mathrm{P}$ & .763 & .198 & .018 & & \\
\hline
\end{tabular}

Note: * indicates significant difference compared with the control group, $\Delta$ indicates significant difference compared with baseline, $\square$ indicates significant difference compared with week 4 endpoint.

\subsection{Impacts of Wisconsin Ginseng on Functional Disability and Quality of Life}

Repeated measure ANOVA were used to compare HAQ-DI of the three groups of participants at different endpoints, the results are shown in Table 4 . The intervention and time interaction effect of the HAQ-DI score were significant $(\mathrm{F}=16.78, \mathrm{P}<0.001)$, therefore, simple effect analysis was further conducted. The results of simple effect analysis show that without considering the treatment effect, there was no significant difference in HAQ-DI scores at different endpoint times in the control group ( $\mathrm{P}>0.05)$, there were significant differences in HAQ-DI scores at different time points in the $3,000 \mathrm{mg}$ low dose and the $6,000 \mathrm{mg}$ high dose groups, further Pairwise comparison of energy point scores in the low dose and high dose groups showed decrease of HAQ-DI scores, as treatment duration increased in time demonstration of a significant decrease of scores was observed; The results of simple effect analysis show that without considering the treatment effect, there was no significant difference in HAQ-DI scores between the three groups at baseline, week 4 endpoints, and week 8 endpoints after treatment $(\mathrm{P}>0.05)$.

The above results showed that HAQ-DI scores in the control group showed no significant change with the duration of treatment, the HAQ-DI scores of the low dose group and the high dose group demonstrated decrease functional disability significantly with the increase of treatment duration, the difference in the intervention effect of the three treatment arms on HAQ-DI did not reached a level of significant $(\mathrm{P}>0.05)$. 
Table 4. Comparison of changes in functional disability using HAQ-DI after specified treatments.

\begin{tabular}{lllllll}
\hline Variable & Group & Week 0 & Week 4 & Week 8 & F & P \\
\hline & Placebo & $0.53 \pm 0.29$ & $0.49 \pm 0.26$ & $0.48 \pm 0.29$ & 3.064 & .081 \\
HAQ-DI & 3,000mg Ginseng & $0.53 \pm 0.12$ & $0.45 \pm 0.12^{\Delta}$ & $0.35 \pm 0.11^{\star}$ & 13.922 & .001 \\
& 6,000mg Ginseng & $0.72 \pm 0.25$ & $0.54 \pm 0.24 \Delta$ & $0.37 \pm 0.18^{\star}$ & 68.286 & .000 \\
& F & 1.030 & .172 & .522 & .604 & \\
& P & .382 & .844 & & \\
\hline
\end{tabular}

Note: $\Delta$ indicates significant difference compared with baseline, $\mathbf{\square}$ indicates significant difference compared with week 4 endpoint.

\subsection{Impacts of Wisconsin Ginseng on Second Messengers}

Repeated measure ANOVA were used to compare cAMP $\mathrm{nmol} / \mathrm{L}$ levels of the three groups of participants at different endpoints, the results are shown in Table 5. The intervention and time interaction effect of the cAMP score were significant $(\mathrm{F}=5.86, \mathrm{P}<0.001)$, therefore, simple effect analysis was further conducted. The results of simple effect analysis show that without considering the treatment effect, there was no significant difference in cAMP levels at different endpoint times in the control group $(\mathrm{P}>0.05)$, there were significant differences in cAMP levels at different time points in the $3,000 \mathrm{mg}$ low dose and the $6,000 \mathrm{mg}$ high dose groups, further Pairwise comparison of $\mathrm{nmol} / \mathrm{L}$ levels in the low dose and high dose groups showed decrease of cAMP levels, as treatment duration increased in time demonstration of a significant decrease of $\mathrm{nmol} / \mathrm{L}$ levels was observed; The results of simple effect analysis show that without considering the treatment effect, there was no significant difference in cAMP levels between the three groups at baseline, week 4 endpoints, and week 8 endpoints after treatment $(\mathrm{P}>0.05)$.

The above results showed that cAMP levels in the control group showed no significant change with the duration of treatment, the cAMP levels of the low dose group and the high dose group demonstrated a decrease of second messengers significantly with the increase of treatment duration, the difference in the intervention effect of the three treatment arms on cAMP levels did not reached a level of significant $(\mathrm{P}>0.05)$.

Table 5. Comparison of second messenger changes using cAMP after specified treatments.

\begin{tabular}{|c|c|c|c|c|c|c|}
\hline Variable & Group & Week 0 & Week 4 & Week 8 & $\mathbf{F}$ & $\mathbf{P}$ \\
\hline \multirow{4}{*}{ cAMP } & Placebo & $156.98 \pm 25.66$ & $153.55 \pm 22.68$ & $154.01 \pm 30.2$ & 0.560 & 0.599 \\
\hline & 3,000mg Ginseng & $155.33 \pm 14.23$ & $150 \pm 12.87 \Delta$ & $140.41 \pm 13.79 \Delta \square$ & 77.119 & 0.001 \\
\hline & $6,000 \mathrm{mg}$ Ginseng & $154.55 \pm 15.81$ & $146.28 \pm 19.17 \Delta$ & $134.99 \pm 18.63 \Delta \mathbf{\square}$ & 29.497 & 0.001 \\
\hline & $\mathrm{P}$ & 0.974 & .869 & .463 & & \\
\hline
\end{tabular}

Note: $\Delta$ indicates significant difference compared with baseline, $\boldsymbol{\bullet}$ indicates significant difference compared with week 4 endpoint.

Repeated measure ANOVA were used to compare cGMP $\mathrm{nmol} / \mathrm{L}$ levels of the three groups of participants at different endpoints, the results are shown in Table 6 . The intervention and time interaction effect of the cGMP levels were not significant $(\mathrm{F}=3.058, \mathrm{P}<0.061)$, the effect of the treatment was not significant $(\mathrm{F}=0.624, \mathrm{P}=0.552)$.

The difference in the intervention effect of the three treatment arms on cGMP levels did not reached a level of significant $(\mathrm{P}>0.05)$.

Table 6. Comparison of second messenger changes using cGMP after specified treatments.

\begin{tabular}{llllll}
\hline Variable & Group & Week 0 & Week 4 & Week 8 & P \\
\hline \multirow{3}{*}{ cGMP } & Placebo & $7.36 \pm 1.71$ & $7.01 \pm 1.49$ & $6.67 \pm 1.33$ & \\
& $3,000 \mathrm{mg}$ Ginseng & $7.17 \pm 1.73$ & $6.71 \pm 1.42$ & $5.41 \pm 1.28$ & 0.241 \\
& $6,000 \mathrm{mg}$ Ginseng & $7.03 \pm 1.7$ & $6.37 \pm 1.24$ & $4.84 \pm 0.79$ \\
\hline
\end{tabular}

\subsection{Comparison of Wisconsin Ginseng Efficacy}

Kruskal-Wallis test was used to compare the efficacy at weeks 4 and 8 endpoints after treatment. The results were shown in Table 7. There was no significant difference in efficacy between the groups at week 4 endpoints after treatment $(\mathrm{Z}=2.914, \mathrm{P}=0.233$ ), while there was a significant difference in efficacy between the groups at week 8 endpoint after the $6,000 \mathrm{mg}$ high-dose group treatment $(\mathrm{Z}=6.753, \mathrm{P}=0.034)$.

Table 7. Comparison of efficacy at weeks 4 and 8 endpoints between the three specified treatments.

\begin{tabular}{|c|c|c|c|c|c|c|c|}
\hline Parameter & Group & Remission & Significantly effective & Effective & Ineffective & $\mathbf{Z}$ & $\mathbf{P}$ \\
\hline \multirow{3}{*}{ Week 4} & Placebo & 0 & 0 & 6 & 2 & \multirow{3}{*}{2.914} & \multirow{3}{*}{0.233} \\
\hline & 3,000mg Ginseng & 0 & 0 & 3 & 1 & & \\
\hline & $6,000 \mathrm{mg}$ Ginseng & 1 & 0 & 4 & 0 & & \\
\hline \multirow{3}{*}{ Week 8} & Placebo & 0 & 0 & 6 & 2 & \multirow{3}{*}{6.753} & \multirow{3}{*}{0.034} \\
\hline & 3,000mg Ginseng & 0 & 1 & 3 & 0 & & \\
\hline & $6,000 \mathrm{mg}$ Ginseng & 2 & 1 & 2 & 0 & & \\
\hline
\end{tabular}




\subsection{Safety}

There were no AEs reported also no difference in complete blood count $(\mathrm{CBC})$, hepatic, and renal function parameters found within and between treatments.

\section{Discussion}

Despite extensive efforts and considerable expense, there is no known effective cure for RA and the fatigue associated leading low quality of life [22]. Past pharmacological studies affirmed that the primary active components of Panax quinquefolius are ginsenosides, polysaccharides, peptides, phytosterols, polyacetylenes and amino acids, which have shown to have a major influence on the non-specific immune system, specific immune system, cellular immunity and humoral immunity, enhancing immunity as an immunomodulator $[23,24,8]$.

Oriental Medicine treatment focus aim is on tonifying the primordial qi and purging pathogenic factors by regulating the function of lung, and kidney which Wisconsin ginseng is an ideal herbal botanical candidate due its immunoregulation and immune substitution properties, with the function of tonifying qi and yin, tonifying the lung and the kidney [25]. To our knowledge, no published study has evaluated Wisconsin ginseng intervention for adult RA patients. The present study represents a double- blind randomized clinical trial (RCT), our goal was to determine efficacy with a secondary aim observe if the treatment was safe and well tolerated. The overall compliance suggests that there were no dosing-related problems across arms and that the standard dose was well tolerated as only 3 patients dropped out post 4 week treatment with no AEs reported which is consistent with past clinical trials unitizing Panax quinquefolius that demonstrated little to none toxicity and AEs $[14,26,27]$. We administered a dose of $3 \mathrm{~g}$ and $6 \mathrm{~g}$ vs placebo, which is the recommended dosage range of Panax quinquefolius in Oriental Medicine [13].

The research data shows Wisconsin ginseng is effective against RA fatigue and inflammation at a dose of 3,000 $\mathrm{mg} /$ day, however noteworthy significant effectiveness was shown at $6,000 \mathrm{mg} /$ day appearing to increase energy, decrease fatigue, inflammation, functional disability, and secondary massagers more than did a placebo, as measured by questionnaire scales and biological observation biomarkers. The VAS-F [28] scale accesses energy and fatigue results of the study after baseline to week 8 demonstrated $3,000 \mathrm{mg} /$ day trial patients energy had increased by $20 \%$ and fatigue decreased by $20 \%$ points, further at $6,000 \mathrm{mg} /$ day trial patients energy had increased $34 \%$ and fatigue decreased by $36 \%$ points, the placebo group energy increased $2 \%$ and fatigue demonstrated decrease $0 \%$ points. HAQ-DI [29] results at $6,000 \mathrm{mg} /$ day demonstrated the most improvement. All participants at baseline had HAQ-DI scores from 0 to 1 which is mild difficulties to moderate disability. After week 8 of treatment all treatment arms remained between 0 and 1 which was consistent throughout the study. The exploratory cAMP and cGMP secondary massagers results of the study after baseline to week 8 demonstrated $6,000 \mathrm{mg} /$ day decreased levels cAMP $19 \% \mathrm{nmol} / \mathrm{L}$ and cGMP $2 \% \mathrm{nmol} / \mathrm{L}$ decrease, results were clinically insignificancy for other treatment arms. It is curious that the participants of all treatment arms baseline DAS28 [30] ESR values were near high disease activity and CRP values were moderate disease severity activity of RA. DAS28 values respectively the $3,000 \mathrm{mg} /$ day treatment arm results by week 8 showed a decrease by ESR 0.98 and CRP 0.84 points which is a moderate improvement. At 6,000 $\mathrm{mg}$ /day treatment arm results by week 8 showed a decrease by ESR $1.87 \%$ and CRP $1.29 \%$ points which is a good improvement. By contrast the placebo results by week 8 showed a decrease by ESR 0.35 and CRP 0.25 points which is no improvement. Clinically meaningful results may not be realized until 2 months after starting the high does Wisconsin ginseng with comparative difference between the groups had statistical significance $(\mathrm{P}<0.01)$.

Given the exploratory nature of the study, The mechanisms by means which Wisconsin ginseng applies pharmacological effect on RA are not known. In spite of the uncertainty encompassing the etiology of rheumatic diseases, chronic inflammation and autoimmunity are regarded to play critical roles in both the prevalence and progression of rheumatic diseases. Ginsengs ginsenosides demonstrate, anti-fatigue, anti-inflammatory, and immunostimulatory properties in preclinical data. Hence Wisconsin ginseng may have a curative effect on the pathogenesis of rheumatic diseases [31, 32]. Albeit little is known regarding the pathophysiology of RA associated fatigue, Substantial objective evidence promoting that ginseng may be effective for ameliorative fatigue in chronic illness derived from preclinical and clinical data. The active compounds of Wisconsin ginseng are conceived to demonstrate curative action on the central nervous system, antioxidant, and anti-inflammatory properties, as well as cortisol-modulating effects. Researchers rationalization for Panax quinquefolius as a curative potential treatment for fatigue associated with chronic illness is grounding in these past findings [14].

The molecular mechanism by which $\mathrm{Rg}^{1}$ and $\mathrm{Rb}^{1}$ regulate immune function can regulate intracellular secondary messengers cGMP and cAMP levels [33, 34]. Wisconsin ginseng ginsenosides reduced secondary messenger cAMP levels which can inhibit the release of central neurotransmitters and promote the release of dopamine [35]. The Yin and Yang theory is opposite and interrelated process of Yang eliminating Yin which is correlated with increased cAMP levels. Wisconsin Ginseng ginsenosides may be the contributing factor for revived the Yin and demonstrated restoration towards the relative balance of Yin and Yang thereby reducing fatigue and inflammation in RA patients of the identification pattern of qi and yin deficiency.

Panax quinquefolius is a herbal botanical that demonstrated through many studies to be beneficial for chronic illness notably fatigue relief. The ginsenoside $R b^{1}$ is a major constituent of both American ginseng and Asian ginseng. 
Ginsengs are more often than not administered orally similar to many other herbal botanical medicines. Once ingested by the gut, ginsenoside $\mathrm{Rb}^{1}$ main metabolic pathway is through degraded by intestinal microbiota after which stepwise transformed into compound $\mathrm{K}$ [36, 37]. Compound $\mathrm{K}$ metabolite of $\mathrm{Rb}^{1}$ is absorbed into the systemic circulation thereby exerting unique biofunctions [38, 39]. Numerous earlier investigations have exhibited the curative properties of ginseng ginsenosides effects for RA pathogenesis. Protective joint effects of compound $\mathrm{K}$ on $\mathrm{RA}$ pathogenesis were examined in prior vitro studies. Previous analysis reveal compound $\mathrm{K}$ considerably diminished the production of matrix metalloproteinases (MMPs). MMPs regulate varied aspects of inflammation and immunity as identified by researchers in MMP biology. MMP-1 is a collagenases and MMP-3 is a stromelysins both are part of the one of the most commonly used groupings MMPs. MMP-1 and MMP-3 which can cleave extracellular matrix components as well as activate other MMPs in RA patients. Compound $\mathrm{K}$ reduced production induced by cytokines, which stimulate cells such as fibroblasts and osteoblasts, and can cause indirect tissue damage and RA fibroblast-like synoviocytes which was achieved by inhibiting janus kinase and extracellular signal-regulated kinase signaling pathways [40]. Previous preclinical data of the impact of ginseng ginsenosides $\mathrm{Rb}^{1}$ on $\mathrm{RA}$ pathogenesis was reviewed. The exploratory data suggested that $\mathrm{Rb}^{1}$ reduced cell infiltration and cartilage destruction in the arthritic joint in the RA animal model reviewed, resulting in significant decrease in TNF- $\alpha$ expression. Based on this and other preclinical data of $\mathrm{Rb}^{1}$ which is a major ginsenoside in Panax quinquefolius we were encouraged that the use in treatment of RA patients could lead curative results in a clinical trial setting [41].

This study has several limitations. A small sample size due to a high attrition rate and the emergence of a global health emergency may be a factor. Lower than anticipated sample size might have led to a type II error. It was not possible to fully control other potential medical causes of fatigue. Additionally, fatigue responses are subject to placebo responses. therefore, some changes in the reported severity of fatigue could have been the result of a placebo effect.

The source of Wisconsin ginseng was well controlled tested by multiple laboratories including the China Food and Drug Administration (CFDA). Despite Panax quinquefolius not being a regulated drug by the Food and Drug Administration (FDA) in the USA there is rigorous EPA and USDA regulations, but there is a likelihood of minor variable proportions of core ginsenosides in different crops from the same region, but major variable proportions of grown in other nations. The cultivation and extraction technology of Panax quinquefolius also the natural growing environment conditions may contribute to the decrease of ginsenosides a variability in efficacy and potentially in safety across batches from nations outside the USA [42, 44] which may result in just as high of a variability in efficacy and potentially in safety across batches.

\section{Conclusion}

This study was the first randomized clinical trial (RCT) with real-world RA patients and suggests that Wisconsin ginseng treatment is a safe alternative for many RA patients. Previous studies demonstrate the herbal botanical Wisconsin ginseng can decrease fatigue and is relatively nontoxic, inexpensive natural product with broad complementary and integrative health medicinal as well as biomedical pharmacological activities.

In conclusion, the present, very preliminary, data suggesting that these results of the concurrent use of Wisconsin ginseng administered as an adjunct to conventional RA treatment might be considered as a safe and valuable complimentary, add-on therapy for the management RA and associated fatigue also did generate favorable effects on inflammation levels. It would be reasonable to consider further investigation with larger sample sizes will be required to adequately address the effectiveness of Wisconsin ginseng in improving the fatigue symptoms of RA patients.

\section{Acknowledgements}

This article would not have been possible without the guidance and financial support from Prof. Xinwei Song Department of Rheumatism and Immunology, Zhejiang Provincial Hospital of Chinese Medicine I am truly grateful.

\section{References}

[1] Is the incidence of rheumatoid arthritis rising? Myasoedova E, Crowson CS, Kremers HM, Therneau TM, Gabriel SE. 6, s.1.: Arthritis Rheum, 2010, Vol. 62. 1576-1582.

[2] Rheumatoid arthritis. Scott DL, Wolfe F, Huizinga TW. 9746, London, England: Lancet, 2010, Vol. 376.

[3] The prevalence and meaning of fatigue in rheumatic disease. Wolfe F, Hawley DJ, Wilson K. s.1.: J Rheumatol, 1996, Vol. 23.

[4] Correlates of fatigue in older adults with rheumatoid arthritis. Belza BL, Henke CJ, Yelin EH, Epstein WV, Gilliss CL. 93-9, s.1.: Nurs Res, 1993, Vol. 42.

[5] The assessment of fatigue. A practical guide for clinicians and researchers. Dittner AJ, Wessely SC, Brown RG. s.l.: J Psychosom Res, 2004, Vol. 56, pp. 157-70.

[6] Genetics of rheumatoid arthritis susceptibility, severity, and treatment response. Viatte S, Barton A. 4, s.1.: Seminars in immunopathology, 2017, Vol. 39, pp. 395-408.

[7] Four different patterns of fatigue in rheumatoid arthritis patients: results of a Q-sort study. Stephanie Nikolaus1, Christina Bode, Erik Taal and Mart A. F. J. van de Laar. s.l.: RHEUMATOLOGY, 2010, Vol. 49, pp. 2191-2199.

[8] Pharmacological and Clinical Efficacy of American Ginseng (Panax Quinquefolius): A Mini Review. andenhouten Eric E., Li Yan, Ying Wang. 4, s.1.: International Journal of Chinese Medicine, 2019, Vol. 3, pp. 64-79. ISSN: 2578-9465 (Print); ISSN: 2578-9473 (Online). 
[9] National Institutes of Health (NIH). The National Center for Complementary and Integrative Health (NCCIH). National Institutes of Health (NIH). [Online] 0926, 2020. [Cited: 09 26, 2020.] https://www.nccih.nih.gov/about/nccih-facts-at-a-glance-and-missio n.

[10] Oriental medicine: an introduction. D., Ehling. 4, s.l.: Ther Health Med., 2001, Vol. 7, pp. 71-82.

[11] Giovanni Maciocia. Diagnosis in Chinese Medicine A Comprehensive Guide. London: Elsevier, 2004. 0-4430-6448-2.

[12] Professor Gao Mingli's clinical experience in treating rheumatoid arthritis with deficiency of qi and Yin. Wu Qihua, Gao Mingli. 1, Shenyang: Rheumatism and Arthritis, 2019, Vol 8. ISSN: 2095-4174.

[13] Gamble, Dan Bensky Steve Clavey Erich Stöger with Andrew. Chinese Herbal Medicine: Materia Medica (Portable 3rd Ed.). s.1.: Eastland Press, 2015. ISBN: 0-939-616-82-3 978-0-939616-82-4.

[14] Ginseng as a Treatment for Fatigue: A Systematic Review. Noe 1 M. Arring, Denise Millstine, Lisa A. Marks, Lillian M. Nail. 00, s.1.: THE JOURNAL OF ALTERNATIVE AND COMPLEMENTARY MEDICINE, 2018, Vol. 00, pp. $1-10$.

[15] United States Department of Agriculture (USDA). Agriculture Marketing Service (USDA). United States Department of Agriculture (USDA). [Online] 08 31, 2020. [Cited: 08 31, 2020.] https://www.ams.usda.gov/grades-standards/ginseng-grades-a nd-standards.

[16] GINSENG BOARD OF WISCONSIN, INC. Why Wisconsin Ginseng? www.ginsengboard.com. [Online] GINSENG BOARD OF WISCONSIN, INC., 08 31, 2020. [Cited: 08 31, 2020.] http:/www.ginsengboard.com/pages/service-style-1/.

[17] Validity and reliability of a scale to assess fatigue. K A Lee, G Hicks, and G Nino-Murcia. 3, s.1.: Psychiatry Res, 1990, Vol. 36.

[18] Clinical measurement of disease activity in rheumatoid arthritis: why, how and utility of patient self-assessment. Peter P Cheung, \& Laure Gossec. 3, s.l.: Int. J. Clin. Rheumatol., 2014, Vol. 9, pp. 327-339. ISSN 1758-4272.

[19] The status and evaluation of the diagnostic and therapeutic criteria of traditional Chinese medicine for rheumatoid arthritis. Bing, Zhao Xinxiu and Qin. s.l.: CHINESE ARCHIVES OF TRADITIONAL CHINESE MEDICINE, 2009, Vol. 9, pp. 1879-1880. ISSN: 1673-7717.

[20] Efficacy of medical ozone-majorautohaemotherapy combined with traditional Chinese medicine fumigation in the treatment of patients with rheumatoid arthritis. Li Shenghong, Chang Guangchi, Zhou Fang, Pang Yanli, Xiao Junzhou, Dong Qingpeng, Yun Xiawang [1]. 6, s.1.: Pain Clinic Journal, 2013, Vol. 9. ISSN: 1672-9633.

[21] Determining rheumatologists' accuracy at assessing functional disability in rheumatoid arthritis patients using the Health Assessment Questionnaire-Disability Index. Carter JD, Lodhi AB, Nagda SR, et al. 5, 2007: J Rheumatol., Vol. 34, pp. 958-63.

[22] Four different patterns of fatigue in rheumatoid arthritis patients: results of a Q-sort study. Nikolaus S, Bode C, Taal E, van de Laar MA. 11, s.1.: Rheumatology (Oxford)., 2010, Vol. 49, pp. 2191-2199.
[23] Research on immunological regulation effect of Radix Panacis Quinquefolii and related preparation. Wang YH, Liu Zhong S, Guan F, Wang R. s.1.: Chin Arch Tradit Chin Med (Chin), 2004, Vol. 22, pp. 566-567.

[24] Korean Red Ginseng exhibits no significant adverse effect on disease activity in patients with rheumatoid arthritis: a randomized, double-blind, crossover study. Cho SK, Kim D, Yoo D, Jang EJ, Jun JB, Sung YK. 2, s.1.: J Ginseng Res., 2018, Vol. 42, pp. 144-148.

[25] Effect of Immune No. 2 on the immune reconstitution in patients with HIV/AIDS after highly active antiretroviral treatment: a randomized double blind placebo controlled clinical trial. Wang J, Li Y, Tang YL, Lin HS, Wu XF, Liu J. 5, s.1.: Chin J Integr Med., 2013, Vol. 19, pp. 340-346.

[26] Efficacy and safety of American ginseng (Panax quinquefolius L.) extract on glycemic control and cardiovascular risk factors in individuals with type 2 diabetes: a double-blind, randomized, cross-over clinical trial. Vuksan V, Xu ZZ, Jovanovski E, et al. 3, s.1.: Eur J Nutr., 2019, Vol. 58, pp. 1237-1245.

[27] Pilot study of Panax quinquefolius (American ginseng) to improve cancer-related fatigue: a randomized, double-blind, dose-finding evaluation: NCCTG trial N03CA. Barton DL, Soori GS, Bauer BA, et al. 2, s.1.: Support Care Cancer., 2010, Vol. 18, pp. 179-187.

[28] Validity and reliability of a scale to assess fatigue. Lee KA, Hicks G, Nino-Murcia G. 3, s.l.: Psychiatry Res., 1991, Vol. 36 , pp. 291-298.

[29] Confirmation of the validity of the HAQ-DI in two populations living with chronic illnesses. Sousa KH, Kwok OM, Ryu E, Cook SW. 1, s.1.: J Nurs Meas., 2008, Vol. 16, pp. 31-42.

[30] Modified disease activity scores that include twenty-eight-joint counts. Development and validation in a prospective longitudinal study of patients with rheumatoid arthritis. Prevoo ML, van 't Hof MA, Kuper HH, van Leeuwen MA, van de Putte LB, van Riel PL. 1, s.l.: Arthritis Rheum., 1995, Vol. 38, pp. 44-48.

[31] Colds and influenza: a review of diagnosis and conventional, botanical, and nutritional considerations. Roxas M, Jurenka J. 1, s.1.: Altern Med Rev, 2007, 2007, Vol. 12, pp. 25-48.

[32] Ameliorative effects of ginseng and ginsenosides on rheumatic diseases. YS., Yi. 3, s.1.: J Ginseng Res., 2019, Vol. 43, pp. 335-341.

[33] Regulatory effects of ginsenosides on lipid metabolism. LV Wen-shan, YANG Li-li, HUANG Hai-tao. 2, 2013: International Journal of Endocrinology and Metabolism (Chin), Vol. 33, pp. 115-117, 125.

[34] STUDIES ON THE MECHANISMS OF IMMUNOREGULATORY EFFECTS OF GINSENOSIDE RGI IN AGED RATS. Zhang, M Liu and JT. 2, s.l.: Acta Pharmaceutica Sinica, 1996, Vol. 31, pp. 95-100.

[35] Effects of Panax ginseng root on the vertical and horizontal motor activities and on brain monoamine-related substances in mice. Itoh T, Zang YF, Murai S, Saito H. 5, s.l.: Planta Med., 1989, Vol. 55, pp. 429-433.

[36] Degradation of ginsenosides in humans after oral administration. Tawab MA, Bahr U, Karas M, Wurglics M, Schubert-Zsilavecz M. 8, s.1.: Drug Metab Dispos., 2003, Vol. 31, pp. 1065-1071. 
[37] Comparative analysis of the gut microbiota in people with different levels of ginsenoside Rb1 degradation to compound $K$. Kim KA, Jung IH, Park SH, Ahn YT, Huh CS, Kim DH. 4, s.1.: PLoS One., 2013, Vol. 8.

[38] Screening of Drug Metabolizing Enzymes for the Ginsenoside Compound K In Vitro: An Efficient Anti-Cancer Substance Originating from Panax Ginseng. Xiao J, Chen D, Lin XX, et al 2, s.1.: PLoS One., 2016, Vol. 11.

[39] Anti-cancer effects of ginsenoside compound $k$ on pediatric acute myeloid leukemia cells.. Chen Y, Xu Y, Zhu Y, Li X. 1, s.1.: Cancer Cell Int., 2013, Vol. 13.

[40] Joint-protective effects of compound $K$, a major ginsenoside metabolite, in rheumatoid arthritis: in vitro evidence. Choi YS, Kang EH, Lee EY, et al. 8, s.l.: Rheumatol Int., 2013, Vol. 33, pp. 1981-1990.
[41] Anti-arthritic effect of ginsenoside Rb1 on collagen induced arthritis in mice. Kim HA, Kim S, Chang SH, Hwang HJ, Choi YN. 10, s.1.: Int Immunopharmacol., 2007, Vol. 7, pp. 1286-1291.

[42] Analysis of main chemical composition of Panax quinquefolium from different habitats. Haibo, Ma Jing and Rui. s.l.: Northwest Pharmaceutical Journal (Chin), 2019, Vol. 5, pp. 601-604.

[43] Study on quality of Panax quinquefolius from different habitats. Tang Liyue, Chen Xiaorong, and Li Wengui. 001, s.l.: Chinese journal of Health Care Nutrition (Chin), 2018, Vol. 028.

[44] Decreasing, null and increasing effects of eight popular types of ginseng on acute postprandial glycemic indices in healthy humans: the role of ginsenosides. Sievenpiper JL, Arnason JT, Leiter LA, Vuksan V. 3, s.1.: J Am Coll Nutr., 2004, Vol. 23, pp. 248-258. 